\title{
Two Cases of Subacute Combined Degeneration: Magnetic Resonance Findings
}

\author{
Susumu Okada ${ }^{1}$, Tomoyuki Kuwako ${ }^{1}$, Hidenobu Nakajoํ, Makiko Ishihara ${ }^{2}$, \\ Fumio Uchiyama ${ }^{3}$, Rinnosuke Obo $^{4}$, Norio Yokose ${ }^{5}$ and Makoto Hamamoto \\ ${ }^{1}$ Department of Radiology, Nippon Medical School Chiba-Hokusoh Hospital \\ ${ }^{2}$ Department of Radiology, Nippon Medical School \\ ${ }^{3}$ Department of Radiology, Ebina-Sogo Hospital \\ ${ }^{4}$ Department of Internal Medicine, Fuchu-Keijinkai Hospital \\ ${ }^{5}$ Department of Internal Medicine, Nippon Medical School Chiba-Hokusoh Hospital
}

\begin{abstract}
We report two cases of subacute combined degeneration. Both patients had undergone total gastrectomy. The chief complaints were numbness in both upper extremities in case 1 and numbness in both the upper and lower extremities and gait disturbance in case 2 . The pain, temperature, and vibration senses of both patients were decreased. Laboratory examinations showed macrocytic anemia and a decreased serum vitamin B12 level in both cases. In both cases T2-weighted magnetic resonance images showed an area of hyperintensity in the dorsal columns of the cervical spinal cord. The patients were treated with vitamin B12. The abnormal signals had disappeared on follow-up magnetic resonance examination 1 year later in case 1 and 3 months later in case 2. These patients showed neurological improvement, but the numbness in the upper extremities persisted even after the area of abnormal signal intensity had disappeared in case 1.
\end{abstract}

(J Nippon Med Sch 2006; 73: 328-331)

Key words: subacute combined degeneration, vitamin B12, magnetic resonance

\section{Introduction}

Vitamin B12 deficiency can induce disorders of the brain, spinal cord, optic nerve, and peripheral nerves ${ }^{1.2}$. Subacute combined degeneration (SCD) of the spinal cord is an uncommon cause of myelopathy but is the most frequent clinical manifestation of vitamin $\mathrm{B} 12$ deficiency. The magnetic resonance (MR) findings of $\mathrm{SCD}$ of the spinal cord have recently been reported in several papers ${ }^{1-8.10}$. We report two cases of SCD with typical MR abnormalities that disappeared on follow-up studies.

\section{Case Reports}

\section{Case 1}

A 71-year-old man complained of numbness of 4 months' duration in both upper extremities. He had undergone total gastrectomy for a gastric ulcer 27

Correspondence to Susumu Okada, Department of Radiology, Nippon Medical School Chiba-Hokusoh Hospital, 1715

Kamagari, Inba-mura, Inba-gun, Chiba 270-1694, Japan

E-mail: okada@nms.ac.jp

Journal Website (http://www.nms.ac.jp/jnms/) 


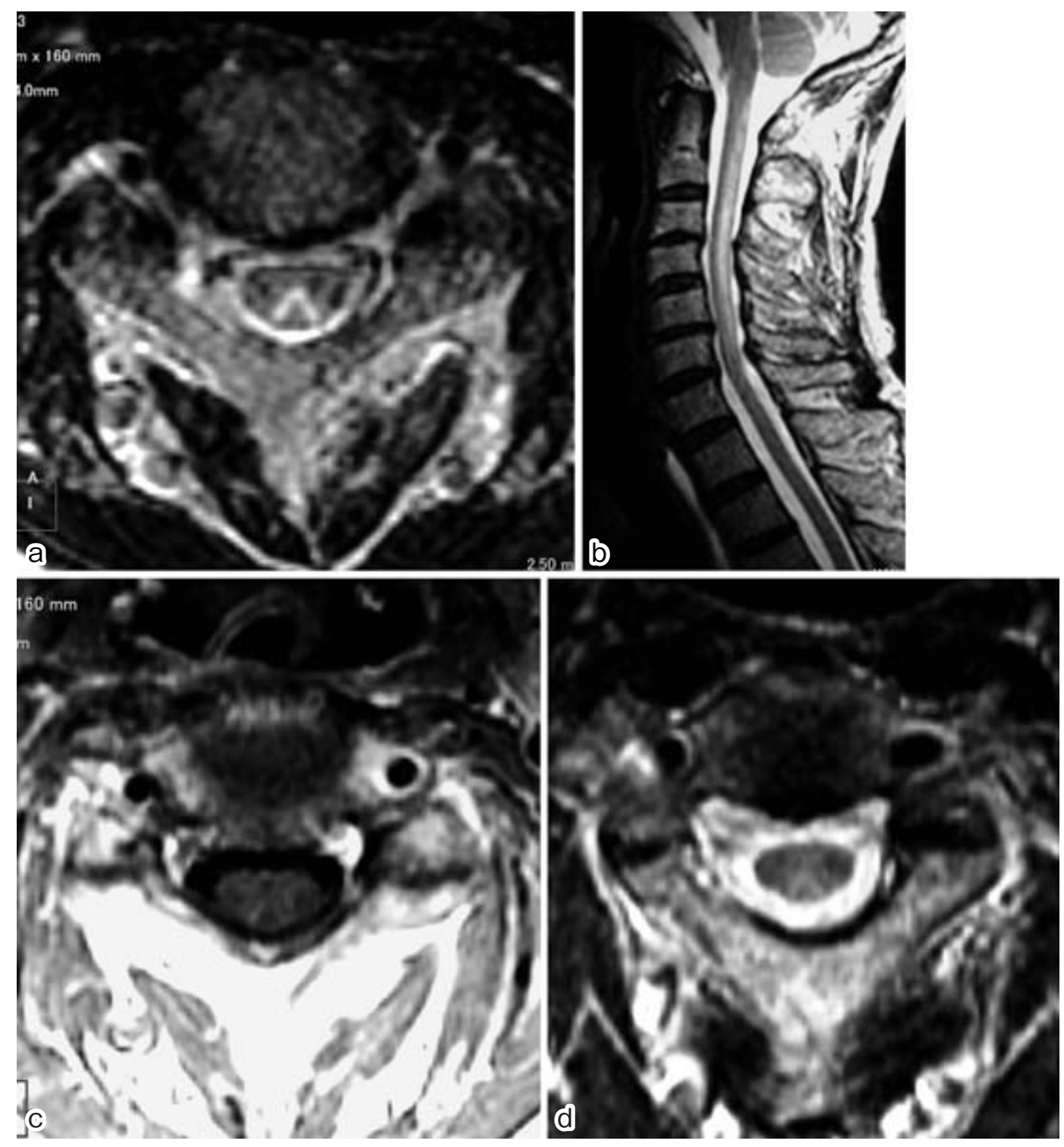

Fig. 1 Case 1: Initial T2-weighted axial image at the C2 level (a) showing an area of hyperintensity in the dorsal columns. Sagittal T2-weighted image (b) showing an area of hyperintensity from level C2 to level C6. This area shows slight enhancement after administration of contrast medium (c). Follow-up MR scan 8 months after the initial study showing a persistent area of hyperintensity less evident on the T2-weighted image (d). This area had disappeared on the 1-year follow-up study (not shown).

years earlier.

Abnormal neurological findings were confined to the upper limbs and consisted of moderately decreased pain and temperature sensation and slightly decreased vibration sense. There was no decrease in light touch sensation. The deep tendon reflexes were normal. Lhermitte's sign, Romberg's sign, and Babinski’s sign were absent.

Laboratory tests revealed macrocytic anemia (red blood cells, $201 \times 10^{4}$; hemoglobin, $9.0 \mathrm{~g} / \mathrm{d}$; hematocrit, 25.7\%; mean corpuscular volume, $127.9 \mathrm{fl}$; mean corpuscular hemoglobin, 44.8 pg; mean corpuscular hemoglobin concentration, $35.0 \mathrm{~g} / \mathrm{d} l$ ), but the white blood cell count and the platelet count were normal. The serum vitamin B12 level was less than $70 \mathrm{pg} / \mathrm{ml}$ (normal, 230 $800 \mathrm{pg} / \mathrm{ml}$ ), and the folic acid level was $28.0 \mathrm{ng} / \mathrm{ml}$ (normal, 2.5 9.2 ng/ $\mathrm{ml}$ ). A bone marrow biopsy revealed megaloblastic changes. A diagnosis of vitamin B12 deficiency was made on the basis of these findings.

The initial MR examination of the cervical spine was performed using a 1.5 - $\mathrm{T}$ unit and showed an 


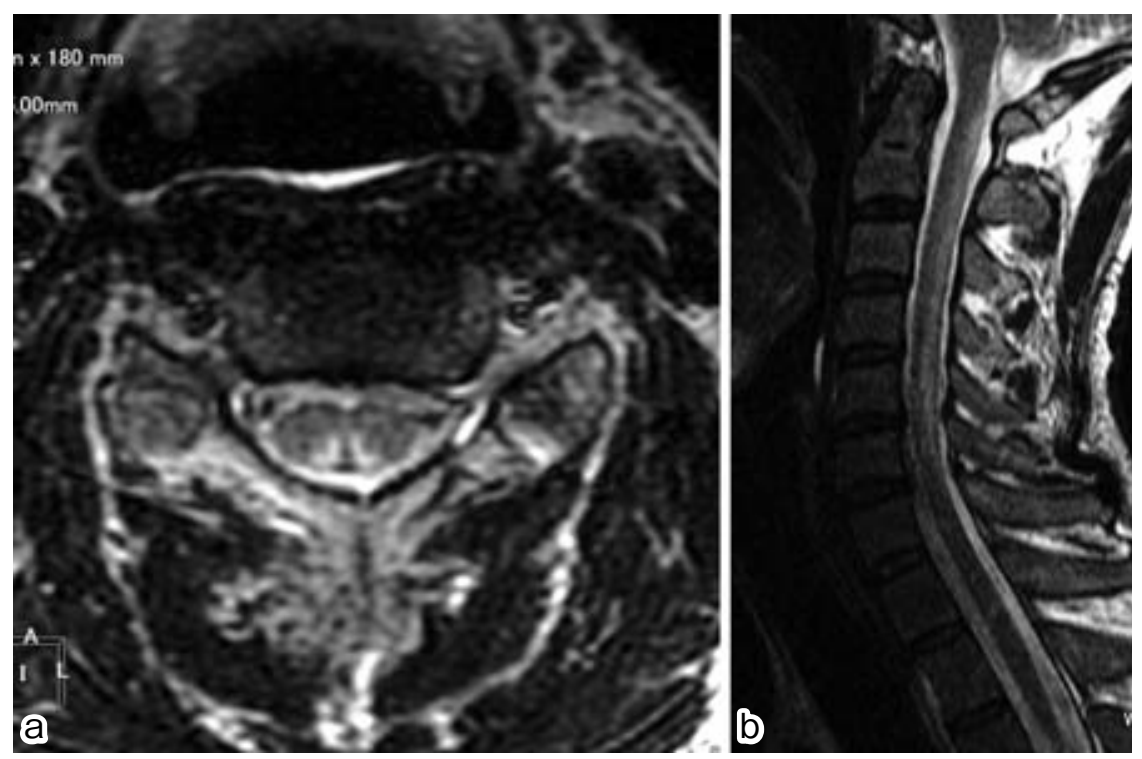

Fig. 2 Case 2: Initial T2-weighted axial image at the C2 level (a) showing an area of hyperintensity in the dorsal columns. Sagittal T2-weighted image (b) showing an area of hyperintensity from level C2 to level C6. This area was not enhanced after administration of contrast medium (not shown).

area of hyperintensity involving the dorsal columns at levels $\mathrm{C} 2$ to $\mathrm{C} 6$ on T2-weighted images (Fig. 1a,

b). The same area was slightly enhanced after administration of gadolinium as a contrast medium (Fig. 1c).

The patient was treated with intramuscular injections of vitamin B12, $1 \mathrm{mg}$ daily for 7 days, and then twice a week for 1 month. There was neurological improvement, but the numbness in the upper extremities persisted. Follow-up MR 1 month after the initial study showed persistent signal change on T2-weighted images, but no contrast enhancement was detected. Weekly injection of 1 $\mathrm{mg}$ of vitamin B12 was continued for 5 years. The T2 signal abnormalities were less evident but remained on the 8-month follow-up MR study (Fig. 1d). The abnormalities had disappeared by the time of the 1-year follow-up study. However, the numbness of the upper extremities remained even after the area of abnormal signal intensity had disappeared.

\section{Case 2}

A 50-year-old man complained of numbness in both the upper and lower extremities and a gait disturbance of 2 months' duration. He had undergone total gastrectomy for gastric cancer 10 years earlier.

Neurological examination showed decreased pain, temperature, and vibration senses in the upper and lower extremities. Light touch sensation was not decreased. Romberg's sign was present. The heelknee sign was present. Deep tendon reflexes were normal. Lhermitte's sign and Babinski’s sign were absent.

Laboratory tests revealed macrocytic anemia (red blood cells, $124 \times 10^{4}$, hemoglobin, $4.8 \mathrm{~g} / \mathrm{d}$; hematocrit, 14.9\%; mean corpuscular volume, $120.0 \mathrm{fl}$; mean corpuscular hemoglobin, 38.7 pg; mean corpuscular hemoglobin concentration, $32.2 \mathrm{~g} / \mathrm{d} l$ ), and the white blood cell count and the platelet count were 2,880 and $3.4 \times 10^{4}$, respectively. The serum vitamin B12 level was $80 \mathrm{pg} / \mathrm{ml}$, and the folic acid level was $12.7 \mathrm{ng} / \mathrm{ml}$. Vitamin B12 deficiency was diagnosed on the basis of these findings.

The initial MR study of the cervical spine showed an area of hyperintensity that involved the dorsal columns at levels $\mathrm{C} 2$ to $\mathrm{C} 6$ on $\mathrm{T} 2$-weighted images (Fig. 2a, b). The area was not enhanced after administration of gadolinium. The radiological studies and measurement of tumor markers showed no evidence of metastasis.

The patient was given vitamin B12, $1.5 \mathrm{mg}$ orally daily for 4 months and thereafter vitamin B12, $1 \mathrm{mg}$, 
daily. Follow-up MR 3 months after the initial study showed no abnormal signals. The patient showed good neurological improvement.

\section{Discussion}

The causes of vitamin B12 deficiency can be divided into three main categories: inadequate intake (strict vegetarians), malabsorption (pernicious anemia, total gastrectomy, etc.) and other conditions (nitrous oxide anesthesia, autoimmune disorders, etc. $)^{3.4}$. Pernicious anemia and total gastrectomy are the most common causes of vitamin B12 deficiency ${ }^{3}$.

The earliest symptoms and signs of vitamin B12 deficiency are paresthesia and areflexia related to peripheral nerve damage. Spinal cord lesions may develop later and are manifested by dysesthesia and impaired deep sensation ${ }^{2}$. In advanced cases, subsequent degeneration of the corticospinal tracts and dorsal columns may lead to paraplegia 2 .

The most consistent MR finding in patients with SCD is increased signal intensity on T2-weighted images in the dorsal columns in the cervical and/or the thoracic portions of the spinal $\operatorname{cord}^{1-9}$. The differential diagnosis of lesions involving the dorsal columns includes multiple sclerosis, infectious or postinfectious myelitis, posterior spinal artery syndrome, malignant lymphoma, traumatic cord injury, and paraneoplastic syndromes ${ }^{2,6,7}$. The areas of hyperintensity on $\mathrm{T} 2$-weighted images in our two cases were consistent with previous reports on SCD but differed from the findings in the diseases listed above. However, copper deficiency myelopathy sometimes has a clinical and radiological presentation similar to that of SCD, and the MR findings may be almost identical to those of SCD ${ }^{9}$. Measurement of the serum copper level should be considered to differentiate SCD from this rare myelopathy. A rare case of SCD that showed anterior column involvement has also been rep-orted ${ }^{10}$; T2weighted MR images in this case showed areas of increased intensity similar to those in multiple sclerosis. In such cases radiological differentiation from multiple sclerosis may be difficult.

The symptoms of SCD may resolve if vitamin B12 therapy is started early in the course of the disease ${ }^{4.6}$. Most symptoms improved in our two patients, but symptoms did not resolve completely in case 1. The abnormal signals in the dorsal columns had disappeared on follow-up MR study 3 months after the initial study in case 2 but they and the symptom of upper-extremity numbness remained even 8 months later in case 1. Abnormal MR findings sometimes persist longer in cases diagnosed at an advanced stage than in cases diagnosed and treated at an early stage. MR may be helpful for both diagnosing SCD and monitoring the response to therapy ${ }^{4,6}$.

\section{References}

1. Fritschi J, Sturzenegger M: Spinal MRI supporting myelopathic origin of early symptoms in unsuspected cobalamin deficiency. Eur Neurol 2003; 49: 146-150.

2. Yamada K, Shrier DA, Tanaka H, Numaguchi Y: A case of subacute combined degeneration: MRI findings. Neuroradiology 1998; 40: 398-400.

3. Katsaros VK, Glocker FX, Hemmer B, Schumacher M: MRI of spinal cord and brain lesions in subacute combined degeneration. Neuroradiology 1998; 40: 716-719.

4. Ravina B, Loevner LA, Bank W: MR findings in subacute combined degeneration of the spinal cord: A case of reversible cervical myelopathy. AJR 2000; 174: 863-865.

5. Pema PJ, Horak HA, Wyatt RH: Myelopathy caused by nitrous oxide toxicity. Am J Neuroradiol 1998; 19: 894-896.

6. Timms SR, Curé JK, Kurent JE: Subacute combined degeneration of the spinal cord: MR Findings. AJNR 1993; 14: 1224-1227.

7. Tajima Y, Mito Y, Owada Y, Moriwaka F, Tashiro K: MR appearance of subacute combined degeneration of the spinal cord. Jpn J Psychiatry Neurol 1994; 48: 611-614.

8. Berlit P, Ringelstein A, Liebig T: Spinal MRI precedes clinical improvement in subacute combined degeneration with B12 deficiency. Neurology 2004; 63: 592-594.

9. Kumar N, Ahlskog JE, Klein CJ, Port JD: Imaging features of copper deficiency myelopathy: a study of 25 cases. Neuroradiology 2006; 48: 78-83.

10. Karantanas AH, Markonis A, Bisbiyiannis G: Subacute combined degeneration of the spinal cord with involvement of the anterior columns: a new MRI finding. Neuroradiology 2000; 42: 115-117.

(Received, August 21, 2006) (Accepted, September 22, 2006) 